\title{
Atypical Resting-State Functional Connectivity of Intra/Inter-Sensory Networks is Related to Symptom Severity in Young Boys with Autism Spectrum Disorder
}

\author{
Jia Wang \\ Harbin Medical University \\ Xiaomin Wang \\ Harbin Medical University \\ Runshi Wang \\ University of Electronic Science and Technology of China \\ Xujun Duan \\ University of Electronic Science and Technology of China \\ Heng Chen \\ School of Medicine,Guizhou University \\ Changchun He \\ University of Electronic Science and Technology of China \\ Jinhe Zhai \\ Harbin Medical University \\ Huafu Chen \\ University of Electronic Science and Technology of China \\ Lijie Wu ( $\square$ wulijiehyd@126.com ) \\ Harbin Medical University
}

\section{Research}

Keywords: autism spectrum disorder, resting-state functional magnetic imaging, rest state network, auditory network, sensorimotor network, visual network

Posted Date: October 7th, 2020

DOl: https://doi.org/10.21203/rs.3.rs-86127/v1

License: (9) This work is licensed under a Creative Commons Attribution 4.0 International License.

Read Full License 
Version of Record: A version of this preprint was published at Frontiers in Physiology on March 24th, 2021. See the published version at https://doi.org/10.3389/fphys.2021.626338. 


\section{Abstract}

Background Autism spectrum disorder (ASD), a neurodevelopmental disorder, has been reported with an altered brain connectivity pattern in the sensory network using resting-state functional magnetic imaging (rs-fMRI) compared to typical developing participants (TDs). However, there is still no consistent conclusion. In the current study, we investigated the alterations of the intra-network and inter-network connectivity pattern relating to sensory in children with ASD compared with TDs, and further assessed whether these alterations are associated with autistic behavioral symptoms.

Methods rs-fMRI was used to assess young boys with ASD ( $N=29)$ and TD $(\mathrm{N}=29)$, aged 3-7-years. Groups were matched for age and handiness. Spatial patterns of the sensory rest state networks (RSNs) were obtained using group Independent component analysis (ICA) method, and between-groups differences were evaluated within each sensory network. Then, the time series of each RSN were extracted from each participant preprocessed data. Correlation analysis was assessed among intra- and inter-network functional connectivity (FC) and symptom severity in children with ASD.

Results Four sensory components were identified, including auditory network (AN), higher visual network (HVN), primary visual network (PVN) and sensorimotor network (SMN). Functional images revealed two sensory networks exhibiting significant increased FCs in ASD group, located within AN and SMN. Higher positive connectivity between PVN and HVN in ASD group is associated with symptom severity.

Conclusion Current study might shed light that the abnormal connectivity patterns of sensory network regions may underlie impaired higher-order multisensory integration in ASD children, and social impairment of ASD are caused probably by aberrant FCs involving inter/intra- sensory network.

\section{Introduction}

Autism spectrum disorder (ASD), a neurodevelopmental disability, is associated with deficits in social communication and social interactions as well as restricted, repetitive patterns of behaviors, interests, and/or activities (American Psychiatric Association, 2013). Recent estimates are that ASD affects 1/59 children aged 8 years old (Baio et al., 2018), which is higher than the prevalence of 1/68 children in 2012 (Christensen et al., 2016) and the estimates of 1/110 children in 2006 (Autism and Developmental Disabilities Monitoring Network Surveillance Year 2006 Principal Investigators, 2009). A recent search for the prevalence of ASD in China found that Jilin City has a similar prevalence of autism in China to that in the West, at approximately $1 \%$, and the results from Shenzhen and Jiamusi cities are also in line with Western estimates (Sun et al., 2019).

Sensory processing differences have long been associated with ASD, and they have recently been added to the diagnostic criteria for the updated diagnostic criteria in the Diagnostic and Statistical Manual of Mental Disorders, 5th Edition (DSM-V) (American Psychiatric Association, 2013). The previous paper integrated the empirical literature on sensory processing in ASD from the last decade,and suggested a high prevalence of sensory symptoms, with reports ranging from 60 to $96 \%$ of children with ASD 
exhibiting some degree of atypical responses to sensory stimuli (Schauder and Bennetto, 2016). Clinical studies of two have indicated that central nervous system factors play a prominent role in abnormal sensory processing in ASD (Hannant et al., 2016, Brandwein et al., 2015). Sensory processing is a very complex process involving the cooperation of different brain regions. Different sensory-related neurons, neuron clusters, or dynamic interactions between brain regions are integrated to form the sensory functional network of the brain. Linear independent component analysis (ICA), a blind source signal separation method, is used to analyze fMRI data. It can extract a series of temporally independent brain activity signals or spatially independent brain networks. In recent years, ICA has been widely used in brain network research and has achieved many important research results, especially in the default mode network (DMN) and saliency network (SN) (Bi et al., 2018, He et al., 2018, Duan et al., 2017, Guo et al., 2018, Green et al., 2016). In the study of sensory network level, ICA found that children with ASD showed decreased FC in the VN and increased FC in the motor network (Ahmadi et al., 2014, Uddin et al., 2013a). However, the recent research suggested that the RSNs with increased FC include the auditory network (AN) and sensory-motor network (SMN) and mixed FC in visual network (VN) (Bi et al., 2018).

Past studies with ASD children have focused on connectivity patterns in a single area, an interesting network. The signals in different brain regions might show a certain correlation in time series even though there may be no structural connections.

The principle of functional integration believes that the function of the brain is achieved through the interaction of multiple brain regions (Aertsen, Gerstein et al. 1989), when one sensory brain region is activated, other sensory brain regions are simultaneously activated or weakened. Therefore, the connection patterns between different sensory brain regions or brain networks reflect higher-order multisensory integration to a certain extent. Individuals with ASD atypically recruit visual brain regions during processing of simple auditory stimuli (Jao Keehn et al., 2017). Rs-fMRI study showed that weaker connectivity between the primary visual cortex and sensorimotor regions in preschool-aged children was correlated with increased sensory hypersensitivity in the visual/auditory domain (Shen et al., 2016). In the study of sensory network, Ahmadi et al. showed that in individuals with ASD, FC levels between the insular-temporal/ACC and the VN, DMN and ACC were decreased; however, FC between the DMN and VN was increased (Ahmadi et al., 2014). Nebel et al. found increased FC between the VN and motor network in ASD school-age children (Nebel et al., 2016). Although there has been a degree of progress in establishing the sensory FC architecture in RSNs in individuals with ASD, a consistent conclusion has not been reached, especially with a lack of evidence in younger ASD children.

As ASD is an early-onset disorder, children with ASD showed early brain overgrowth in the early stages of life (Courchesne et al., 2001; Sparks et al., 2002), among autistic boys, 7-year-olds had the largest whole brain volume (Courchesne et al., 2001). In the study of fMRI, Nomi et al. found that children with ASD aged 11 and under, compared with age-matched TD children, exhibited hyperconnectivity within largescale brain networks (Nomi and Uddin, 2015). Uddin et al. used rs-fMRI and found that hyperconnectivity may be more characteristic of young children with ASD, while hypoconnectivity may begin to emerge in adolescence and persist into adulthood (Uddin et al., 2013b). These previous mixed hyper-and 
hypoconnectivity results suggest that brain FC presents periodical features with increased age. According to the findings of structural and functional imaging, we focused on a narrow age range of 3-7 years in order to understand the neurobiological changes. Besides, we limited the study to male children to minimize developmental differences between different age groups and sexes (Carper et al., 2002, Bloss and Courchesne, 2007). The current study was motivated to examine sensory RSN dysregulation. We hypothesize that the children with ASD would exhibit atypical FC pattern involving inter/intra-sensory networks assessed using ICA. Sensory processing impairment, and associated brain abnormalities, have been shown to be highly correlated with patient outcome in social/emotional functioning, cognitive abilities (Corina \& Singleton, 2009). We further tested whether atypical FC pattern changes of ASD could be a source of the abnormal sensory behavior and social impairments and repetitive patterns of behavior observed in children with ASD.

\section{Materials And Methods}

\section{Participants}

In total, 29 male children with ASD were recruited from the Children Development and Behavioral Research Center of Harbin Medical University, and 29 age- and handedness-matched male TDs were recruited from local kindergartens (Table 1). None of the children had a reported history of any severe medical problem or any neurological or psychiatric condition (with the exception of ASD), and none of the children were taking psychotropic medications. Written informed consent was obtained from the guardians of each subject prior to examination. This study was approved by the ethics review committee of Harbin Medical University. 
Table 1

Demographic data (means \pm SD) of ASD and TD group

\begin{tabular}{|c|c|c|c|c|}
\hline & ASD $(n=29)$ & $\operatorname{TD}(n=29)$ & $t / \chi^{2}$ & $\mathbf{p}$ \\
\hline Age(years, $x \pm s)$ & $4.93 \pm 1.30$ & $4.99 \pm 1.01$ & $-0.206^{1}$ & 0.837 \\
\hline Handiness(right/left) & $22 / 7$ & $26 / 3$ & $1.933^{2}$ & 0.164 \\
\hline $\operatorname{PPVT}(x \pm s)$ & $\begin{array}{l}61.24 \pm \\
17.09\end{array}$ & $\begin{array}{l}99.69 \pm \\
26.93\end{array}$ & $-6.492^{1}$ & $0.000 *$ \\
\hline Mean FD_scrubbing & $\begin{array}{l}0.129 \pm \\
0.045\end{array}$ & $\begin{array}{l}0.136 \pm \\
0.037\end{array}$ & $-0.707^{1}$ & 0.483 \\
\hline AQ total score & $84.19 \pm 1.56$ & $\mathrm{~N} / \mathrm{A}$ & $\mathrm{N} / \mathrm{A}$ & $\mathrm{N} / \mathrm{A}$ \\
\hline \multicolumn{5}{|l|}{ AQ sub-scale } \\
\hline Social skills & $16.42 \pm 4.41$ & $\mathrm{~N} / \mathrm{A}$ & $\mathrm{N} / \mathrm{A}$ & N/A \\
\hline Attention switching & $15.38 \pm 3.98$ & $\mathrm{~N} / \mathrm{A}$ & N/A & $\mathrm{N} / \mathrm{A}$ \\
\hline Attention to detail & $15.62 \pm 6.26$ & $\mathrm{~N} / \mathrm{A}$ & N/A & $\mathrm{N} / \mathrm{A}$ \\
\hline Communication & $19.31 \pm 4.66$ & $\mathrm{~N} / \mathrm{A}$ & $\mathrm{N} / \mathrm{A}$ & $\mathrm{N} / \mathrm{A}$ \\
\hline Imagination & $17.46 \pm 3.69$ & $\mathrm{~N} / \mathrm{A}$ & $\mathrm{N} / \mathrm{A}$ & $\mathrm{N} / \mathrm{A}$ \\
\hline \multicolumn{5}{|l|}{ ADOS sub-scale } \\
\hline Communication & $6.15 \pm 1.79$ & $\mathrm{~N} / \mathrm{A}$ & $\mathrm{N} / \mathrm{A}$ & $\mathrm{N} / \mathrm{A}$ \\
\hline Social interaction & $9.50 \pm 2.30$ & $\mathrm{~N} / \mathrm{A}$ & $\mathrm{N} / \mathrm{A}$ & $\mathrm{N} / \mathrm{A}$ \\
\hline \multicolumn{5}{|l|}{ social interaction } \\
\hline Stereotyped behaviors and restricted interests & $2.15 \pm 1.19$ & $\mathrm{~N} / \mathrm{A}$ & $\mathrm{N} / \mathrm{A}$ & $\mathrm{N} / \mathrm{A}$ \\
\hline \multicolumn{5}{|l|}{ ADIR sub-scale } \\
\hline Social Interaction & $22.24 \pm 3.53$ & $\mathrm{~N} / \mathrm{A}$ & $\mathrm{N} / \mathrm{A}$ & $\mathrm{N} / \mathrm{A}$ \\
\hline Communication & $15.00 \pm 4.31$ & $\mathrm{~N} / \mathrm{A}$ & $\mathrm{N} / \mathrm{A}$ & $\mathrm{N} / \mathrm{A}$ \\
\hline $\begin{array}{l}\text { Restricted, Repetitive, and Stereotyped } \\
\text { Behaviors }\end{array}$ & $7.00 \pm 2.55$ & $\mathrm{~N} / \mathrm{A}$ & $\mathrm{N} / \mathrm{A}$ & $\mathrm{N} / \mathrm{A}$ \\
\hline SRS total score & $88.74 \pm 2.06$ & $\mathrm{~N} / \mathrm{A}$ & $\mathrm{N} / \mathrm{A}$ & $\mathrm{N} / \mathrm{A}$ \\
\hline
\end{tabular}

ASD, autism spectrum disorder; TD, typical developing; PPVT, Peabody Picture Vocabulary Test; ADOS, Autism Diagnostic Observation Schedule; ADI-R, Autism Diagnostic Interview-Revised; N/A, not applicable (TDs did not receive the AQ, SSP, SRS, ADOS, and ADI-R ${ }^{1}$ indicates $t$-test; ${ }^{2}$ indicates chisquare test; ${ }^{*}$ indicates statistical significance $p<0.001$ 


\begin{tabular}{|c|c|c|c|c|}
\hline & ASD $(n=29)$ & $\mathrm{TD}(\mathrm{n}=29)$ & $t / \chi^{2}$ & $\mathbf{p}$ \\
\hline \multicolumn{5}{|l|}{ SRS sub-scale } \\
\hline Social perception & $4.89 \pm 6.08$ & $\mathrm{~N} / \mathrm{A}$ & $\mathrm{N} / \mathrm{A}$ & N/A \\
\hline Social cognition, & $18.85 \pm 4.94$ & N/A & $\mathrm{N} / \mathrm{A}$ & N/A \\
\hline Social communication, & $34.22 \pm 8.39$ & N/A & $\mathrm{N} / \mathrm{A}$ & N/A \\
\hline Social motivation, & $15.48 \pm 4.72$ & N/A & $\mathrm{N} / \mathrm{A}$ & N/A \\
\hline Autism behavior pattern & $15.30 \pm 5.33$ & N/A & N/A & N/A \\
\hline SSP total scale & $\begin{array}{l}147.82 \pm \\
20.63\end{array}$ & N/A & N/A & N/A \\
\hline \multicolumn{5}{|l|}{ SSP sub-scale } \\
\hline Tactile sensitivity & $30.91 \pm 4.12$ & $\mathrm{~N} / \mathrm{A}$ & $\mathrm{N} / \mathrm{A}$ & $\mathrm{N} / \mathrm{A}$ \\
\hline Taste/Smell Sensitivity & $17.27 \pm 2.84$ & $\mathrm{~N} / \mathrm{A}$ & $\mathrm{N} / \mathrm{A}$ & $\mathrm{N} / \mathrm{A}$ \\
\hline Movement Sensitivity & $12.00 \pm 2.79$ & N/A & $\mathrm{N} / \mathrm{A}$ & $\mathrm{N} / \mathrm{A}$ \\
\hline Underresponsive/ & $27.64 \pm 4.37$ & $\mathrm{~N} / \mathrm{A}$ & N/A & $\mathrm{N} / \mathrm{A}$ \\
\hline \multicolumn{5}{|l|}{ Seeks Sensation } \\
\hline Auditory Filtering & $18.55 \pm 4.57$ & $\mathrm{~N} / \mathrm{A}$ & N/A & $\mathrm{N} / \mathrm{A}$ \\
\hline Low Energy/weak & $22.50 \pm 5.57$ & $\mathrm{~N} / \mathrm{A}$ & N/A & $\mathrm{N} / \mathrm{A}$ \\
\hline Visual/Auditory Sensitivity & $18.96 \pm 4.12$ & $\mathrm{~N} / \mathrm{A}$ & $\mathrm{N} / \mathrm{A}$ & $\mathrm{N} / \mathrm{A}$ \\
\hline \multicolumn{5}{|c|}{$\begin{array}{l}\text { ASD, autism spectrum disorder; TD, typical developing; PPVT, Peabody Picture Vocabulary Test; ADOS } \\
\text { Autism Diagnostic Observation Schedule; ADI-R, Autism Diagnostic Interview-Revised; N/A, not } \\
\text { applicable (TDs did not receive the AQ, SSP, SRS, ADOS, and ADI-R }{ }^{1} \text { indicates } t \text {-test; }{ }^{2} \text { indicates chi- } \\
\text { square test; }{ }^{*} \text { indicates statistical significance } p<0.001\end{array}$} \\
\hline
\end{tabular}

\section{Diagnosis And Clinical Assessment}

The ASD diagnosis was based on the DSM-5 (American Psychiatric Association, 2013) combined with the Autism Diagnostic Interview-Revised (ADI-R) (Lord et al., 1994) and Autism Diagnostic Observation Schedule (ADOS) (Lord et al., 2000). In addition, the overall intelligence level of all children was estimated by the Peabody Picture Vocabulary Test (PPVT) (Dunn, 2006). The subjects with ASD were assessed via the Short Sensory Profile (SSP) (Mclntosh DN et al., 1999), a useful screening instrument that is commonly used by occupational therapists to evaluate sensory processing difficulties in children. The Social Responsiveness Scale (SRS) (Constantino, 2002) was also applied to estimate their ability to engage in emotionally appropriate social interactions. The Autism Spectrum Quotient Children's Version 
(AQ-child) (Baron-Cohen et al., 2001) is suitable for screening 4 11-year-old children with autism. The items in this scale were divided into 5 dimensions, including social skills, attention switching, attention to detail, communication, and imagination. Each item was scored with $0 \sim 3$ points and 4 grades. The total score on the scale was $0 \sim 150$. The higher the total score was, the more serious the autistic symptoms were.

\section{Neuroimaging Acquisition}

The rs-fMRI data were collected using a 3.0 T Achieva Magnetic Resonance System (Philips, The Netherlands) at the Department of MR Diagnosis of the affiliated hospital of Harbin Medical University. All MRI scans were performed under sedation using $50 \mathrm{mg} / \mathrm{kg}$ chloral hydrate following a strict clinical protocol. Rs-fMRI images were acquired with a gradient-echo echo-planar pulse: repetition time $(T R)=$ 2,000 ms, echo time $(T E)=30 \mathrm{~ms}, 39$ axial slices, flip angle $=90^{\circ}$, slice thickness $/ \mathrm{gap}=3.0 \mathrm{~mm} / 1.0 \mathrm{~mm}$, field of view $=240 \times 240 \mathrm{~mm}^{2}$, and voxel size $=3.75 \times 3.75 \times 4 \mathrm{~mm}^{3}$. A total of 210 volumes $(7 \mathrm{~min})$ were obtained for each participant.

\section{fMRI data preprocessing}

Data preprocessing was conducted with the Data Processing Assistant for Resting-State fMRI toolbox (DPARSF advanced edition v4.3, http://rfmri.org/DPARSF) (Chao-Gan and Yu-Feng, 2010). The steps included the following: (1) removing the first 10 images to ensure steady-state longitudinal magnetization; (2) slice timing correction; (3) head motion correction with 6 rigid parameters (maximum head motion was less than $2 \mathrm{~mm}$ and 2 degrees for all children); (4) normalization to warp images into standard Montreal Neurological Institute (MNI) space at the resolution of $3 \times 3 \times 3 \mathrm{~mm}^{3}$; and (5) smoothing with a Gaussian kernel (full-width at half-maximum (FWHM) $=6 \mathrm{~mm}$ ) to avoid introducing artificial local spatial correlations.

\section{Independent component analysis (ICA)}

ICA was performed with the Group ICA application of the fMRI Toolbox (GIFTv3.0, http://mialab.mrn.org/software/gift/index.html\#) (Calhoun et al., 2001). The total number of independent components (ICs) was identified by a preliminary dimension, which was estimated in accordance with the minimum description length criterion (Li et al., 2007). There were three further stages: (1) reducing the data dimension by principal component analysis; (2) estimating ICs by using the infomax algorithm; and (3) reconstructing in reverse the IC time series and spatial map of individual subjects (Calhoun et al., 2001, Bell and Sejnowski, 1995). Firstly, the components related to artifacts were discarded upon visual inspection of spatial patterns. Then, four components which are related to sensory networks were selected and represented to be functionally relevant RSN templates in the GIFT toolbox, which were used as provided by previous studies (Beckmann et al., 2005, Damoiseaux et al., 2006). The four components from all subjects were selected by the maximum spatial correlation between ICs and the corresponding template (Liao et al., 2010). In the current study, the four templates were the AN, SMN, primary visual network (PVN), and higher visual network (HVN). 


\section{Intranetwork Connectivity Analysis}

We obtained the spatial pattern of the RSNs, which were the spatial maps of each RSN, across all the subjects and assessed using a one-sample $t$-tests approach $(p<0.001$, false discovery rate (FDR) corrected). Then, between-group differences within each network were obtained using two-sample twotailed $t$-tests (voxel level $p<0.001$, cluster level $p<0.05$, AlphaSim corrected), with comparisons between groups limited to the voxels within each corresponding RSN pattern.

\section{Internetwork Connectivity Analysis}

To investigate between-group differences in the FC between different RSNs identified from ICA, we extracted the time series of each RSN from each participant's preprocessed data, with age, FD, handedness and PPVT scores as covariates. Then, Pearson correlation analysis was used to calculate the correlation coefficients $(r)$ for the time series in each pair of four RSNs, and the value of $r$ was normalized by using Fisher's $r$ to $z$ transformation. For each participant, a $4 \times 4$ FC matrix was obtained. Using a two-sample two-tailed $t$-test approach, we compared the FC values between the ASD and TD groups. We used effect size to measure the strength of the relationship between the ASD and TD groups. The significance level was set at $p<0.05$ (FDR corrected).

\section{Behavioral correlations with intra/internetwork connectivity in the ASD group}

The present study explored whether altered FC was associated with the severity of symptoms in ASD. Pearson correlation analysis was utilized to determine the relationship between the FC values of the atypical RSNs (controlling for the covariates, including age, mean frame displacement (FD) and handedness) and the ADI-R, ADOS, SSP, SRS and AQ scores in the ASD group. A nonparametric Spearman's rank correlation analysis was performed to assess the associations between FC values of the atypical RSNs and the SSP-level scores.

\section{Result}

\section{Resting-state networks}

The number of ICs identified by ICA was forty-six. Four components were identified based on the highest spatial correlation with the RSN templates described previously, including auditory network (AN, 34th component, include bilateral superior temporal gyrus, bilateral middle temporal gyrus, left inferior temporal gyrus, bilateral caudate nucleus, left anterior cingulate and paracingulate gyri, and left precuneus), higher visual network (HVN, 40th component, include bilateral calcarine fissure and surrounding cortex, right lingual gyrus, right inferior occipital gyrus, left middle occipital gyrus, bilateral precuneus, right Inferior frontal gyrus, opercular part, right middle frontal gyrus, right superior frontal gyrus, dorsolateral, right inferior frontal gyrus, triangular part, bilateral precentral gyrus, bilateral postcentral gyrus, right supramarginal gyrus, and left median cingulate and paracingulate gyri), primary visual network (PVN, 13th component, include bilateral lingual gyrus, bilateral calcarine fissure and 
surrounding cortex, left middle occipital gyrus, bilateral median cingulate and paracingulate gyri, left inferior parietal but supramarginal and angular gyri, left angular gyrus and left superior parietal gyrus) and sensorimotor network (SMN, 26th component, include bilateral cerebellum, bilateral precentral gyrus, bilateral postcentral gyrus, bilateral supplementary motor area, bilateral paracentral lobule). A typically spatial pattern in each RSN were obtained by using one-sample t-tests, which was consistent with previous studies (Liao et al., 2010, Zhang et al., 2015, Ding et al., 2011) $(p<0.001$, FDR corrected) (Fig. 1). Between-group differences in intra-network connectivity

Using Two-sample two-tailed $t$-tests, we found the differences in intra-network connectivity between ASD group and TD group. Compared to TD, children with ASD showed significantly increased FC in the AN (left superior temporal gyrus, Brodmann's area: 48 , cluster size: 75 , MNI: $(-42,-18,-6))$ and SMN (right paracentral lobule, Brodmann's area: 4, cluster size: 27 , MNI: $(6,-34,70)$ ) (voxel level $p<0.001$, cluster level $p<0.05$ AlphaSim corrected). (Fig. 2)

\section{Between-group differences in inter-network connectivity}

Significant differences in FC patterns were found by comparing the correlation coefficients in the FC between ASD and TD groups. Compared to TDs, children with ASD showed significantly increased positive FC between AN and SMN ( $p=0.022$, uncorrected), $\mathrm{PVN}$ and $\mathrm{HVN}(p=0.041$, FDR corrected) (Fig. 3).

\section{Relationship with ASD Symptoms}

In our study, we explore the relationship between aberrant intra-network connectivity and the AQ scores of the children with ASD. The results showed significant $(p<0.05)$ positive correlations between the increased auditory network connectivity and the attention switching subscale of AQ. The social skill subscale and imagination subscales of $A Q$ in children with ASD showed a significant positive correlation with increased sensorimotor network connectivity (Fig. 4A). In addition, the aberrant inter-network connectivity between higher visual network and primary visual network showed significant positive correlations with attention switching subscale, imagination subscale and total score of $A Q$, as well as language and communication, communication and social interaction subscales of ADOS (Fig. 4B)

\section{Discussion}

The present study found significant differences in FC at the network level between ASD boys aged 37 years and matched TDs. First, functional images revealed individuals with ASD showed increased FC in the AN and SMN, respectively. Second, when performing an internetwork functional connection analysis, we found significantly higher positive connectivity between the PVN and HVN in the ASD group. Third, the strength of such connections was associated with clinical scale scores, further demonstrating the robustness of our findings.

\section{Altered intranetwork FC in ASD children}


We found that the left superior temporal gyrus (STG) in the AN had an excessive level of FC. Prior structural imaging studies had detected increased gray matter volume in the left STG across different life stages in ASD patients, including toddlers (Xiao et al., 2014), children (Retico et al., 2016), and adolescents (Lim et al., 2015). These findings in the left STG were in accordance with our previous structural neuroimaging study in 3- to 7-year-old ASD boys (Wang et al., 2017). A prominent view is that children with ASD develop excessive brain growth at 2-4 years of age (Courchesne et al., 2001, Sparks et al., 2002), and the development of the auditory cortex does not follow the normal growth epoch (Xiao et al., 2014, Retico et al., 2016, Lim et al., 2015). The theory of synaptic pruning proposed that there was a decreased synaptic pruning in autistic children and adolescents in auditory area (Tang et al., 2014). Combined excessive brain growth and decreased synaptic pruning, children with ASD may have redundant synaptic connections in the immature auditory cortex. Nomi et al. further reported that changes in synaptic density may be related to changes in FC (Nomi and Uddin, 2015). The above findings further provided reliable evidence for increased FC in the auditory area. Correlation analysis with clinical scales found the excessive connectivity in the AN in children with ASD was positively correlated with attention switching of $\mathrm{AQ}(r=0.44, p=0.023)$. The correlation reflected that the excessive connections and increased energy consumption in the AN might affect the children with ASD can not effectively process the auditory information, and show a certain disruption in the ability to switch attention such that "I frequently get so strongly absorbed in one thing that I lose sight of other things", "In a social group, I can easily keep track of several different people's conversations" (Baron-Cohen et al., 2001).

Our study reported that the children with ASD had a significantly stronger FC pattern in the right paracentral lobule of the SMN. Within the sensorimotor brain region, there was greater FC in the bilateral precentral and postcentral gyri in the ASD group than the TD group (Uddin et al., 2013a). Atypical FC in the sensorimotor region of ASD patients has been repeatedly reported by previous studies, although with mixed findings (Uddin et al., 2013a, Anderson et al., 2011, Mostofsky et al., 2009). When using the functional 'connectedness' method and recurrent-seek strategy in the early stages of life, it was also found that ASD children were overconnected in the sensorimotor brain area (Chen et al., 2018), indicating that the ASD children may have abnormal function in the SMN. Atypical brain anatomy and neurodevelopment will inevitably lead to functional changes, with the evidence from behavioral studies further providing a wide range of sensory-motor dysfunction in patients with ASD (Mosconi and Sweeney, 2015, Whyatt and Craig, 2013). In the current study, the hyperconnectivity of the paracentric lobules in the SMN of the ASD children was more prominent. We hypothesized that sensorimotor dysfunction in children with ASD may be associated with dysfunction of the paracentral lobule, combined with the overlapping pattern of activity in the paracentral lobules in the task state (You et al., 2013, Gu et al., 2015). Martino et al. suggested that the paracentral lobule may be associated with abnormalities in the sense of a bodily self that is encased in the body observed in autistic patients (Di Martino A. et al., 2009). Additionally, sensorimotor dysfunction may play a key role in the impairments in social function in ASD populations (Hannant et al., 2016). Interestingly, this hypothesis was confirmed in the exploration of further associations with the AQ scale; that is, the excessive connections in the SMN (paracentral lobule) of those with ASD was significantly positively correlated with social skills and imagination $(r=0.39, p=$ 
$0.046 ; r=0.46, p=0.018)$. The association between the two indicates that the paracentral lobule processes information contributing to social dysfunction in patients with ASD, and its internal abnormal connections may be related to the pathological mechanisms underlying ASD.

\section{Altered internetwork FC in ASD children}

Autistic individuals often exhibit enhanced perceptual abilities when engaged in visual search, visual discrimination, and embedded figure detection tasks (Samson et al., 2012). A recent study showed that local and global visual perception was incomplete in young children with ASD (Jobs et al., 2018). Visual information is transmitted through the conduction pathway. The primary visual pathways included the $P$ (the ventral stream) and M pathways (the dorsal stream) (Tobimatsu and Celesia, 2006, Yamasaki et al., 2014, Yamasaki et al., 2013). Evidence from visual evoked potentials has shown that dysfunctional activity in the P-color pathway in ASD (Fujita et al., 2011). In addition to findings from electrophysiological methods, RSN research finds ASD individuals showed that hyper-connectivity in visual cortex at initial link step distances (Martinez et al. 2019). At the same time, in the research of more complex visual networks, children with ASD showed decreased FC in the VN (Ahmadi et al., 2014). However, the recent research suggested that the RSNs with mixed FC pattern in VN (Bi et al., 2018). In addition to the overall exploration, the visual brain regions/networks are further divided into primary and higher visual brain regions/networks. In the study of visual brain regions, the findings of robust and extensive overconnectivity in the striate (primary visual cortex) and extrastriate visual cortex (higher visual cortex) was intriguing in view of potential local biases in visual perception, supported thus far mainly by findings from behavioral studies (Dakin and Frith, 2005, Mottron et al., 2006). In the study of visual brain networks,children with ASD showed increased connectivity within the occipital pole network and decreased connectivity within the lateral visual network (Xu et al. 2019). To our knowledge, only few rs-fMRI studies have examined FC between primary visual and higher visual regions in young children with ASD. When performing internetwork functional connection analyses, we found significantly higher positive connectivity between the PVN and HVN in the ASD group, even though we did not find abnormal FC in the ASD children in the PVN or HVN, respectively. The aberrant connection patterns were positively correlated with attention switching, imagination, and total score of the $\mathrm{AQ}$ scale and abnormal communication in the ADOS $(r=0.42, p=0.031 ; r=0.45, p=0.021 ; r=0.41, p=0.03 ; r=0.58, p=0.0015$, respectively). We hypothesis that the brain may not reasonably select and distinguish effective information and further communicate with higher visual networks, these might promote children with ASD to pay too much attention to primary visual signals, which is associated with dysfunction of communication.

\section{Limitation}

Our study had some limitations. First, our sample in this study was small and limited to young male children. It was worth exploring whether the research results are generalized to adolescents, adults and female ASD patients. Second, future studies will go further expand the sample and divide it into different subgroups according to the different types and degrees of abnormal sensory behavior emerged by ASD 
children, and explore pertinently the relationship between the abnormal brain function networks related to sensory and specific clinical characterization. Third, current study did not mention or measure power, other characteristics of children including socioeconomic indicators. Fourth,intranetwork connectivity with networks outside primary sensory networks was not examined. Fifth, in this young age range, a pediatric template may be more appropriate. Finally, owing to their younger age, all children cannot finish their scanning without sedation. Thus, all subjects were under sedation using chloral hydrate.

\section{Conclusion}

To conclude, the current findings provide evidence for overconnectivity in sensory networks in young children with ASD. As we found abnormal intra-network connectivity in SMN, and inter-network connectivity between PVN and HVN, the findings further support the notion that deficient/impaired auditory and visual sensory network connectivity patterns may underlie the social dysfunction of ASD. This indicate that therapeutic interventions might target sensory related brain network.

\section{List Of Abbreviations}

ASD: Autism spectrum disorder; rs-fMRI: resting-state functional magnetic imaging; TDs: typical developing participants; RSNs: rest state networks; ICA: Independent component analysis; FC: functional connectivity; AN: auditory network; HVN: higher visual network; PVN: primary visual network; and SMN: sensorimotor network; DMN: default mode network ; STG: superior temporal gyrus; ADI-R: Autism Diagnostic Interview-Revised; ADOS: Autism Diagnostic Observation Schedule; PPVT: Peabody Picture Vocabulary Test; SSP: Short Sensory Profile; SRS: Social Responsiveness Scale; AQ-child: Autism Spectrum Quotient Children's Version; MNI: Montreal Neurological Institute; ICs: independent components.

\section{Declarations}

\section{Ethics approval and consent to participate}

This study was approved by the ethics review committee of Harbin Medical University (Approval no. 2013004). All participants gave written consent.

\section{Consent for publication}

No applicable

\section{Availiability of data and materials}

The datasets generated and/or analyzed during the current study are not publicly available due to specifications on data availability within ethics approval.

\section{Competing interests}


The authors declare that the research was conducted in the absence of any commercial or financial relationships that could be construed as a potential conflict of interest.

\section{Funding}

This study was funded by Natural Science Foundation of China (81874270, 61673089, 81871432, U1808204), China Post-doctoral Science Foundation of China (2018M631962) and the Natural Science Foundation Heilongjiang Province China (QC2018106)

\section{Authors'contrributions}

JW conceived of the study, performed the data acquisition, and drafted and revised the manuscript. XMW was involved in the writing and revision of the manuscript, and contributed to the acquisition of clinical data. RSW was responsible for the analysis and interpretation of imaging data and helped to draft the manuscript. XJD and $\mathrm{HC}$ revised it and made instructive recommendations. LJW and HFC conceived of the study, and helped to received to revise the manuscript. All authors read and approved the final manuscript.

\section{Acknowledgements}

The authors express special thanks to all the children and their parents who participated.

\section{References}

Aertsen AM, Gerstein GL, Habib MK, Palm G. Dynamics of neuronal firing correlation: modulation of "effective connectivity". J Neurophysiol. 1989;61:900-917. doi:10.1152/jn.1989.61.5.900

Ahmadi, S. M. M. , Mohajeri, N. , \& Soltanian-Zadeh, H. . (2015). Connectivity abnormalities in autism spectrum disorder patients: A resting state fMRI study. Electrical Engineering (ICEE) 2014, Iranian Conference. IEEE.1878-1882.

American Psychiatric Association. The diagnostic and statistical manual of mental disorders: DSM 5 (p. 991). Arlington, VA: American Psychiatric Publishing.2013.

Anderson JS, Druzgal TJ, Froehlich A, et al. Decreased interhemispheric functional connectivity in autism. Cereb Cortex. 2011;21:1134-1146. doi:10.1093/cercor/bhq190

Autism and Developmental Disabilities Monitoring Network Surveillance Year 2006 Principal Investigators. Prevalence of autism spectrum disorders - Autism and Developmental Disabilities Monitoring Network, United States, 2006. MMWR Surveill Summ, 2009,58, 1-24.

Baio J, Wiggins L, Christensen DL, et al. Prevalence of Autism Spectrum Disorder Among Children Aged 8 Years - Autism and Developmental Disabilities Monitoring Network, 11 Sites, United States, 2014 [published correction appears in MMWR Morb Mortal Wkly Rep. 2018 May 18;67(19):564] [published 
correction appears in MMWR Morb Mortal Wkly Rep. 2018 Nov 16;67(45):1280]. MMWR Surveill Summ. 2018;67:1-23. Published 2018 Apr 27. doi:10.15585/mmwr.ss6706a1

Baron-Cohen S, Wheelwright S, Skinner R, Martin J, Clubley E. The autism-spectrum quotient (AQ): evidence from Asperger syndrome/high-functioning autism, males and females, scientists and mathematicians [published correction appears in J Autism Dev Disord 2001 Dec;31(6):603]. J Autism Dev Disord. 2001;31:5-17. doi:10.1023/a:1005653411471

Beckmann C F , Deluca M , Devlin J T , et al. Investigations into resting-state connectivity using Independent Component Analysis[J]. Philosophical Transactions of The Royal Society B Biological ences, 2005, 360:1001-1013.

Bell AJ, Sejnowski TJ. An information-maximization approach to blind separation and blind deconvolution. Neural Comput. 1995;7:1129-1159. doi:10.1162/neco.1995.7.6.1129

Bi XA, Zhao J, Xu Q, Sun Q, Wang Z. Abnormal Functional Connectivity of Resting State Network Detection Based on Linear ICA Analysis in Autism Spectrum Disorder. Front Physiol. 2018;9:475. Published 2018 May 8. doi:10.3389/fphys.2018.00475

Bloss CS, Courchesne E. MRI neuroanatomy in young girls with autism: a preliminary study. J Am Acad Child Adolesc Psychiatry. 2007;46:515-523. doi:10.1097/chi.0b013e318030e28b

Brandwein AB, Foxe JJ, Butler JS, Frey HP, Bates JC, Shulman LH, Molholm S. Neurophysiological indices of atypical auditory processing and multisensory integration are associated with symptom severity in autism. J Autism Dev Disord. 2015 ,45:230-44. doi: 10.1007/s10803-014-2212-9.

Calhoun VD, Adali T, Pearlson GD, Pekar JJ. A method for making group inferences from functional MRI data using independent component analysis. Hum Brain Mapp. 2001,14:140-51. doi: 10.1002/hbm.1048.

Carper RA, Moses P, Tigue ZD, Courchesne E. Cerebral lobes in autism: early hyperplasia and abnormal age effects. Neuroimage. 2002,16:1038-51. doi: 10.1006/nimg.2002.1099.

Chao-Gan Y, Yu-Feng Z. DPARSF: A MATLAB Toolbox for "Pipeline" Data Analysis of Resting-State fMRI. Front Syst Neurosci. 2010;4:13. doi:10.3389/fnsys.2010.00013

Chen H, Wang J, Uddin LQ, Wang X, Guo X, Lu F, Duan X, Wu L, Chen H. Aberrant functional connectivity of neural circuits associated with social and sensorimotor deficits in young children with autism spectrum disorder. Autism Res. 2018,11:1643-1652. doi: 10.1002/aur.2029.

Christensen DL, Baio J, Van Naarden Braun K, et al. Prevalence and Characteristics of Autism Spectrum Disorder Among Children Aged 8 Years-Autism and Developmental Disabilities Monitoring Network, 11 Sites, United States, 2012 [published correction appears in MMWR Morb Mortal Wkly Rep. 2016;65(15):404]. MMWR Surveill Summ. 2016;65(3):1-23. Published 2016 Apr 1. doi:10.15585/mmwr.ss6503a1 
Constantino, J.N. The Social Responsiveness Scale. Los Angeles: Western Psychological Services. 2002.

Corina D, Singleton J. Developmental social cognitive neuroscience: insights from deafness. Child Dev.2009,80:952-67. doi: 10.1111/j.1467-8624.2009.01310.x.

Courchesne E, Karns CM, Davis HR, et al. Unusual brain growth patterns in early life in patients with autistic disorder: an MRI study. Neurology. 2001;57:245-254. doi:10.1212/wnl.57.2.245

Courchesne E, Karns CM, Davis HR, et al. Unusual brain growth patterns in early life in patients with autistic disorder: an MRI study. Neurology. 2001;57:245-254. doi:10.1212/wnl.57.2.245

Damoiseaux JS, Rombouts SA, Barkhof F, et al. Consistent resting-state networks across healthy subjects. Proc Natl Acad Sci U S A. 2006;103:13848-13853. doi:10.1073/pnas.0601417103

Ding JR, Liao W, Zhang Z, et al. Topological fractionation of resting-state networks. PLoS One. 2011;6:e26596. doi:10.1371/journal.pone.0026596

Duan $\mathrm{X}$, Chen $\mathrm{H}, \mathrm{He} \mathrm{C}$, et al. Resting-state functional under-connectivity within and between large-scale cortical networks across three low-frequency bands in adolescents with autism. Prog Neuropsychopharmacol Biol Psychiatry. 2017;79:434-441. doi:10.1016/j.pnpbp.2017.07.027

Dunn, L., Dunn, L. M., Arribas, D. PPVT-III Peabody, Test de Vocabulario en Imágenes. Madrid: TEA Ediciones.2006.

Fujiya T, Yamasaki T, Kamio Y, Hirose S, Tobimatsu S. Parvocellular pathway impairment in autism spectrum disorder: Evidence from visual evoked potentials. Research in Autism Spectrum Disorders. 2011,5:277-285.

Green SA, Hernandez L, Bookheimer SY, Dapretto M. Salience Network Connectivity in Autism Is Related to Brain and Behavioral Markers of Sensory Overresponsivity. J Am Acad Child Adolesc Psychiatry. 2016;55:618-626.e1. doi:10.1016/j.jaac.2016.04.013

Gu X, Eilam-Stock T, Zhou T, et al. Autonomic and brain responses associated with empathy deficits in autism spectrum disorder. Hum Brain Mapp. 2015;36:3323-3338. doi:10.1002/hbm.22840

Guo X, Duan X, Suckling J, et al. Partially impaired functional connectivity states between right anterior insula and default mode network in autism spectrum disorder. Hum Brain Mapp. 2019;40:1264-1275. doi:10.1002/hbm.24447

Hannant P, Tavassoli T, Cassidy S. The Role of Sensorimotor Difficulties in Autism Spectrum Conditions. Front Neurol. 2016;7:124. doi:10.3389/fneur.2016.00124

He C, Chen Y, Jian T, et al. Dynamic functional connectivity analysis reveals decreased variability of the default-mode network in developing autistic brain. Autism Res. 2018;11(11):1479-1493. 
Jao Keehn RJ, Sanchez SS, Stewart CR, et al. Impaired downregulation of visual cortex during auditory processing is associated with autism symptomatology in children and adolescents with autism spectrum disorder. Autism Res. 2017;10:130-143. doi:10.1002/aur.1636

Nilsson Jobs E, Falck-Ytter T, Bölte S. Local and Global Visual Processing in 3-Year-Olds With and Without Autism. J Autism Dev Disord. 2018;48:2249-2257. doi:10.1007/s10803-018-3470-8

Li YO, Adali T, Calhoun VD. Estimating the number of independent components for functional magnetic resonance imaging data. Hum Brain Mapp. 2007;28:1251-1266. doi:10.1002/hbm.20359

Liao $\mathrm{W}$, Chen $\mathrm{H}$, Feng Y, et al. Selective aberrant functional connectivity of resting state networks in social anxiety disorder. Neuroimage. 2010;52:1549-1558. doi:10.1016/j.neuroimage.2010.05.010

Lim L, Chantiluke K, Cubillo Al, et al. Disorder-specific grey matter deficits in attention deficit hyperactivity disorder relative to autism spectrum disorder. Psychol Med. 2015;45:965-976.

doi:10.1017/S0033291714001974

Lord C, Risi S, Lambrecht L, et al. The autism diagnostic observation schedule-generic: a standard measure of social and communication deficits associated with the spectrum of autism. J Autism Dev Disord. 2000;30:205-223.

Lord C, Rutter M, Le Couteur A. Autism Diagnostic Interview-Revised: a revised version of a diagnostic interview for caregivers of individuals with possible pervasive developmental disorders. J Autism Dev Disord. 1994;24:659-685. doi:10.1007/BF02172145

Martínez K, Martínez-García M, Marcos-Vidal L, et al. Sensory-to-Cognitive Systems Integration Is Associated With Clinical Severity in Autism Spectrum Disorder. J Am Acad Child Adolesc Psychiatry. 2020;59:422-433. doi:10.1016/j.jaac.2019.05.033

Di Martino A, Ross K, Uddin LQ, Sklar AB, Castellanos FX, Milham MP. Functional brain correlates of social and nonsocial processes in autism spectrum disorders: an activation likelihood estimation metaanalysis. Biol Psychiatry. 2009;65:63-74. doi:10.1016/j.biopsych.2008.09.022

Mcintosh DN, Miller LJ, Shyu V. Short Sensory Profile. In Sensory Profile user's manual. USA: The psychologycal Corporation.1999,59-73.

Mosconi MW, Sweeney JA. Sensorimotor dysfunctions as primary features of autism spectrum disorders. Sci China Life Sci. 2015;58:1016-1023. doi:10.1007/s11427-015-4894-4

Mostofsky SH, Powell SK, Simmonds DJ, Goldberg MC, Caffo B, Pekar JJ. Decreased connectivity and cerebellar activity in autism during motor task performance. Brain. 2009;132:2413-2425.

doi:10.1093/brain/awp088

Page 17/22 
Mottron L, Dawson M, Soulières I, Hubert B, Burack J. Enhanced perceptual functioning in autism: an update, and eight principles of autistic perception. J Autism Dev Disord. 2006;36:27-43. doi:10.1007/s10803-005-0040-7

Nebel MB, Eloyan A, Nettles CA, et al. Intrinsic Visual-Motor Synchrony Correlates With Social Deficits in Autism. Biol Psychiatry. 2016;79:633-641. doi:10.1016/j.biopsych.2015.08.029

Nomi JS, Uddin LQ. Developmental changes in large-scale network connectivity in autism. Neuroimage Clin. 2015;7:732-741. Published 2015 Mar 6. doi:10.1016/j.nicl.2015.02.024

Retico A, Giuliano A, Tancredi R, et al. The effect of gender on the neuroanatomy of children with autism spectrum disorders: a support vector machine case-control study. Mol Autism. 2016;7:5. doi:10.1186/s13229-015-0067-3

Samson F, Mottron L, Soulières I, Zeffiro TA. Enhanced visual functioning in autism: an ALE metaanalysis. Hum Brain Mapp. 2012;33:1553-1581. doi:10.1002/hbm.21307

Schauder KB, Bennetto L. Toward an Interdisciplinary Understanding of Sensory Dysfunction in Autism Spectrum Disorder: An Integration of the Neural and Symptom Literatures. Front Neurosci. 2016;10:268. Published 2016 Jun 17. doi:10.3389/fnins.2016.00268

Shen MD, Li DD, Keown CL, et al. Functional Connectivity of the Amygdala Is Disrupted in Preschool-Aged Children With Autism Spectrum Disorder. J Am Acad Child Adolesc Psychiatry. 2016;55:817-824. doi:10.1016/j.jaac.2016.05.020

Sparks BF, Friedman SD, Shaw DW, et al. Brain structural abnormalities in young children with autism spectrum disorder. Neurology. 2002;59:184-192. doi:10.1212/wnl.59.2.184

Sun X, Allison C, Wei L, et al. Autism prevalence in China is comparable to Western prevalence. Mol Autism. 2019;10:7. doi:10.1186/s13229-018-0246-0

Tang G, Gudsnuk K, Kuo SH, et al. Loss of mTOR-dependent macroautophagy causes autistic-like synaptic pruning deficits Neuron. 2014;83:1131-1143. doi:10.1016/j.neuron.2014.07.040

Tobimatsu S, Celesia GG. Studies of human visual pathophysiology with visual evoked potentials. Clin Neurophysiol. 2006 Jul;117:1414-33. doi: 10.1016/j.clinph.2006.01.004.

Uddin LQ, Supekar K, Lynch CJ, et al. Salience network-based classification and prediction of symptom severity in children with autism. JAMA Psychiatry. 2013;70:869-879.

doi:10.1001/jamapsychiatry.2013.104

Uddin LQ, Supekar K, Menon V. Reconceptualizing functional brain connectivity in autism from a developmental perspective. Front Hum Neurosci. 2013;7:458. doi:10.3389/fnhum.2013.00458 
Wang J, Fu K, Chen L, Duan X, Guo X, Chen H, Wu Q, Xia W, Wu L, Chen H. Increased Gray Matter Volume and Resting-State Functional Connectivity in Somatosensory Cortex and their Relationship with Autistic Symptoms in Young Boys with Autism Spectrum Disorder. Front Physiol. 2017,8:588. doi:

10.3389/fphys.2017.00588.

Whyatt C, Craig C . Sensory-motor problems in Autism, Frontiers in Integrative Neuroscience. 2013,7:51.

Xiao Z, Qiu T, Ke X, et al. Autism spectrum disorder as early neurodevelopmental disorder: evidence from the brain imaging abnormalities in 2-3 years old toddlers. J Autism Dev Disord. 2014;44:1633-1640. doi:10.1007/s10803-014-2033-x

Xu S, Li M, Yang C, et al. Altered Functional Connectivity in Children With Low-Function Autism Spectrum Disorders. Front Neurosci. 2019;13:806. doi:10.3389/fnins.2019.00806

Yamaskai T, Fujita T, Kamio Y, Tobimatau S. Electrophysiological Assessment of Visual Function in Autism Spectrum Disorders. Neuroscience \& Biomedical Engineering. 2013,1(1).

Yamasaki T, Maekawa T, Takahashi H, Fujita T, Kamio Y, Tobimatsu S. Electrophysiology of Visual and Auditory Perception in Autism Spectrum Disorders. Springer New York. 2014.

You X, Norr M, Murphy E, et al. Atypical modulation of distant functional connectivity by cognitive state in children with Autism Spectrum Disorders. Front Hum Neurosci. 2013;7:482.

doi:10.3389/fnhum.2013.00482

Zhang Y, Liu F, Chen H, et al. Intranetwork and internetwork functional connectivity alterations in posttraumatic stress disorder. J Affect Disord. 2015;187:114-121. doi:10.1016/j.jad.2015.08.043

\section{Figures}

\section{Spatial pattern of resting-state networks}

AN
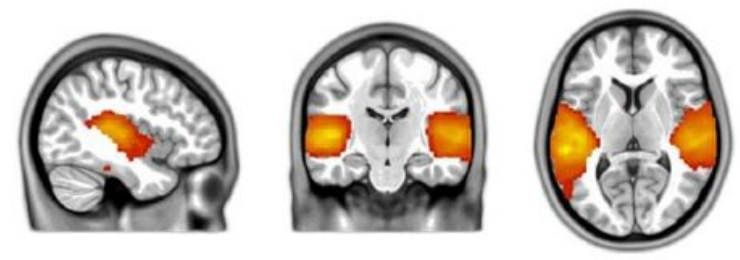

HVN
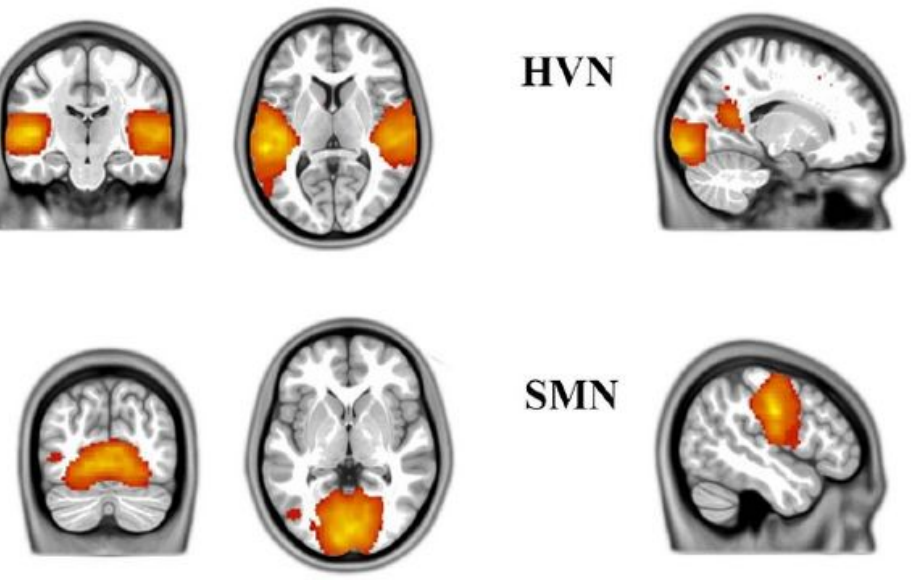

SMN

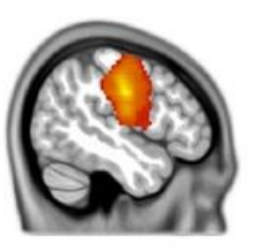

SMN
T value
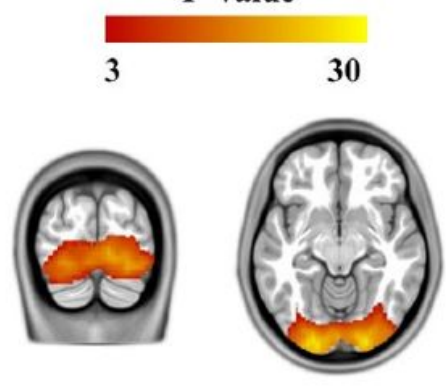

PVN
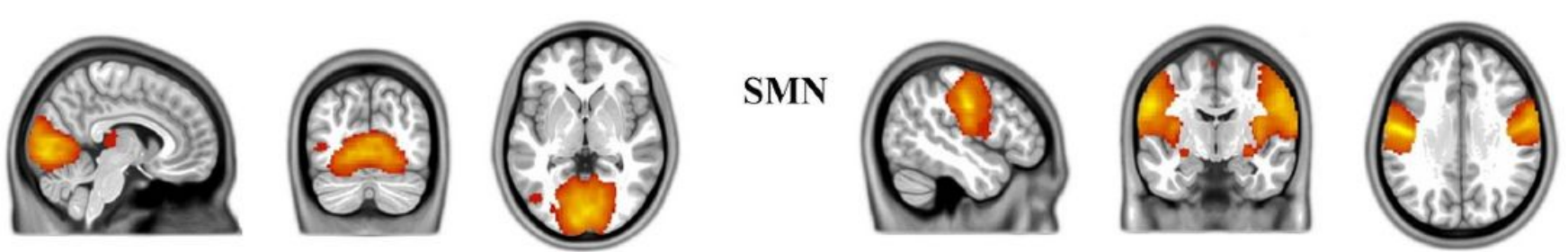
Figure 1

Four brain networks were determined by an independent component analysis. The color bar represents $T$ value in each RSN ( $p<0.001$, FDR corrected). AN, auditory network; HVN, higher visual network; PVN, primary visual network; SMN, sensorimotor network

\section{Altered intra-network connectivity in RSN}

$$
x=-42
$$

AN

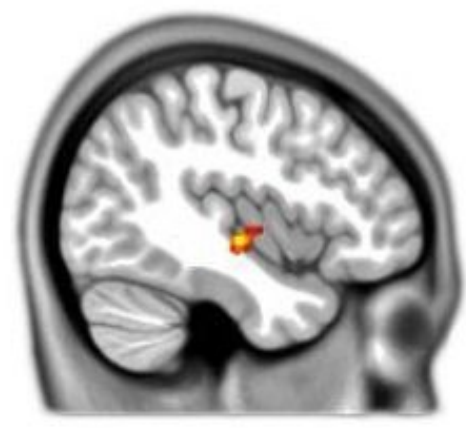

$$
x=6
$$

SMN

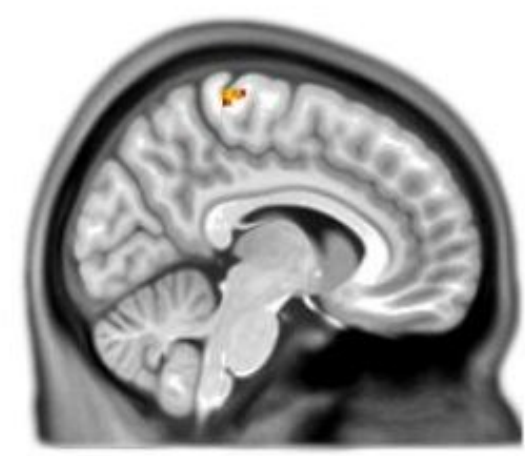

$$
y=-18
$$
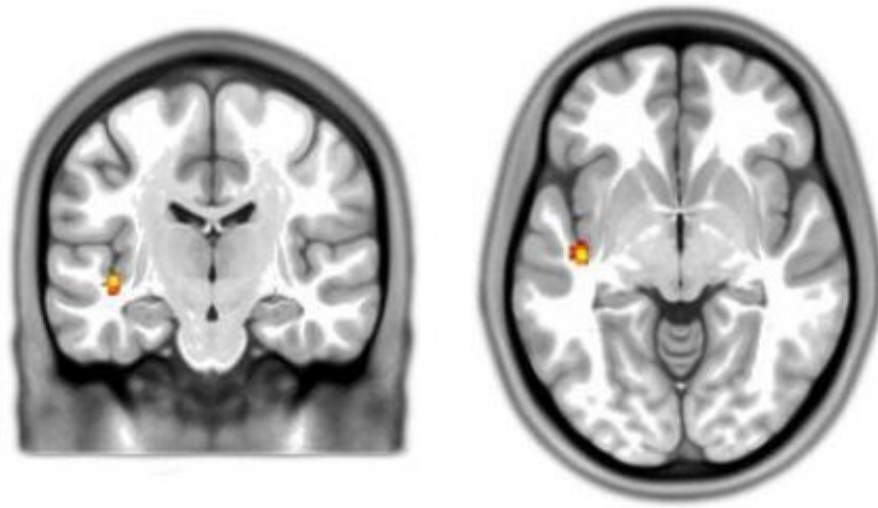

$\mathbf{z}=\mathbf{7 0}$

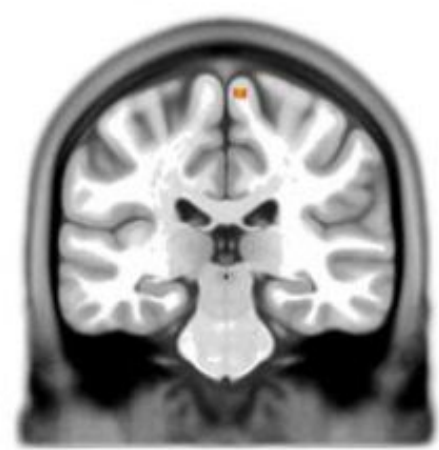

$\mathbf{z}=-6$

$$
y=-34
$$

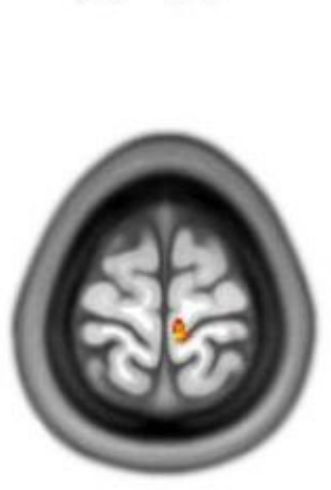

T value

5.02
T value

4.03

3.24

Figure 2

Brain regions of between-group significant differences in intra-network functional connectivity. The yellow color denotes the region with significantly increased connectivity with AN and SMN in ASD group. The color bar represents $T$ value (voxel level $p<0.001$, cluster level $p<0.05$ AlphaSim corrected). AN, auditory network; SMN, sensorimotor network. 
Altered internetwork connectivity beeween ASD group and TD group

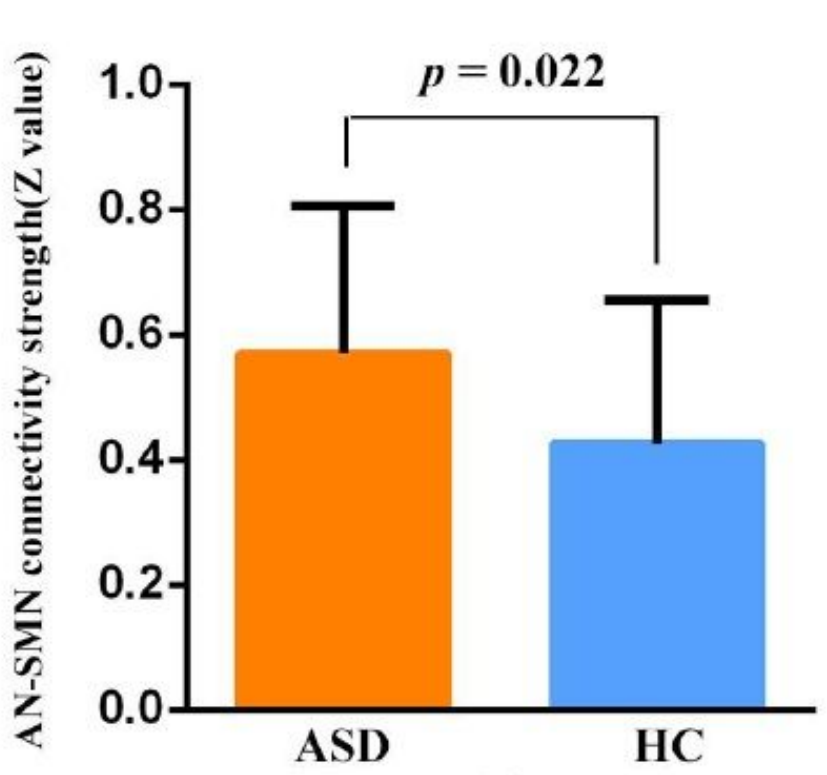

(a)

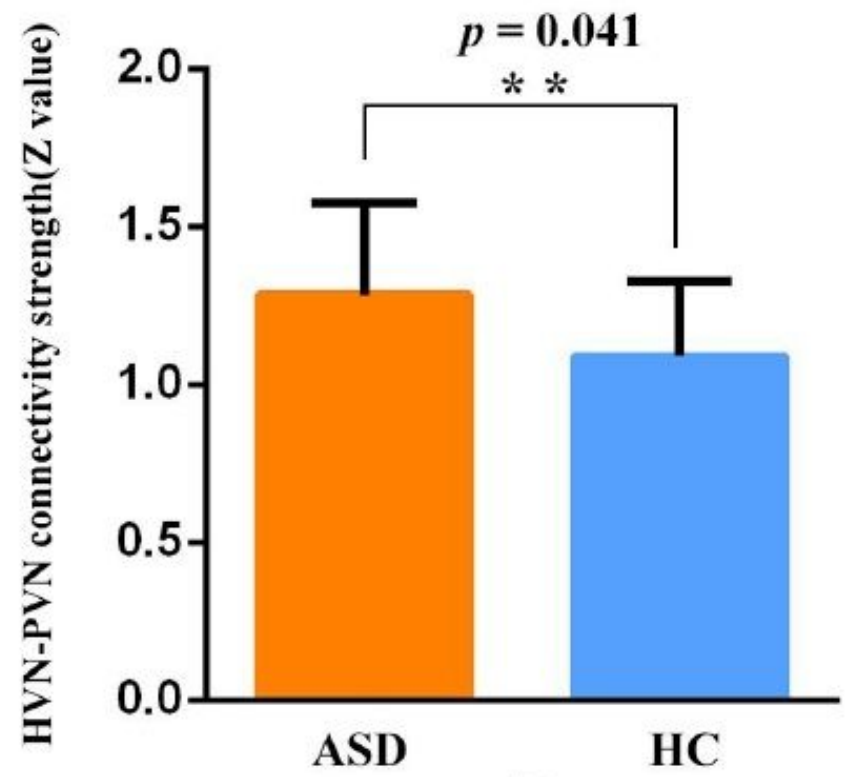

(b)

Figure 3

Connectivity strength ( $z$ value) between two networks in two participant groups. ** represent significant differences after FDR correction. (a) The increased positive FC was found between AN and SMN in ASD group relative to TD ( $p=0.022$, uncorrected). (b) The increased positive FC was found between PVN and HVN in ASD group relative to TD ( $p=0.041$, FDR corrected). Error bars represents standard error of mean. 
(A) Behavioral correlations with Intra- network connectivity in the ASD group
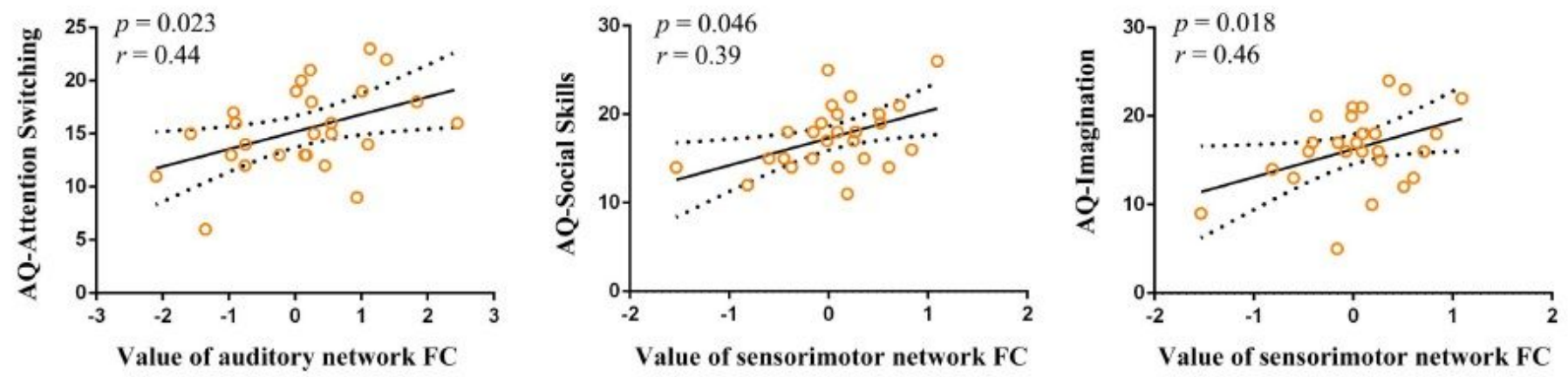

(B) Behavioral correlations with Inter- network connectivity in the ASD group
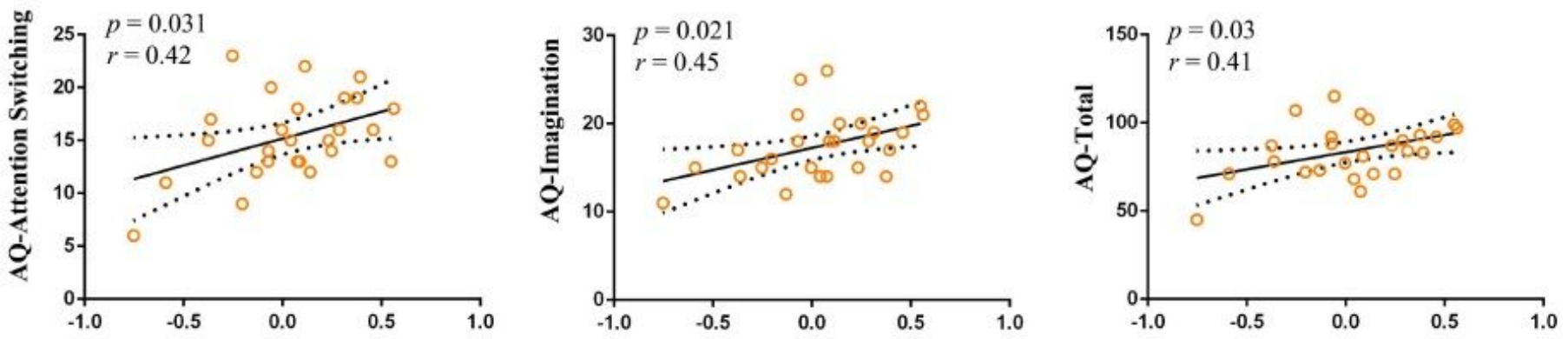

$\mathrm{Z}$ value of PVN-HVN functional connectivity

$\mathrm{Z}$ value of PVN-HVN functional connectivity

$\mathrm{Z}$ value of PVN-HVN functional connectivity
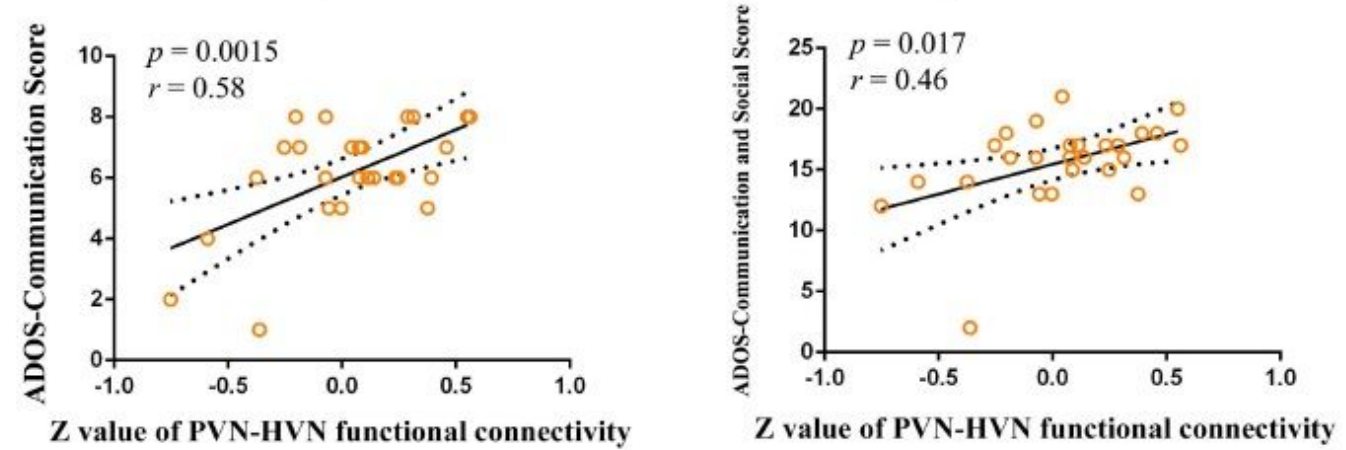

\section{Figure 4}

Correlations between variance in functional connectivity and behavioral scores. (A) Behavioral correlations with intra-network connectivity in ASD group. Scatter plots show significant $(p<0.05)$ positive correlations between aberrant intra-network connectivity and AQ scores. (B) Behavioral correlations with inter-network connectivity in ASD group. Scatter plots show significant $(p<0.05)$ positive correlations between mean $Z$ values of aberrant inter-network connectivity and AQ scores, ADOS scores. AQ, Autism Spectrum Quotient Children's Version; FC, functional connectivity; ADOS, Autism Diagnostic Observation Schedule. 